\title{
Topical Tacrolimus Brings Remission of Vitiligo and Halo Nevus in A Boy: A Case Report
}

\section{Delwar Hossain \\ Shirin Fatema ${ }^{2}$ \\ ASM Mostaque Ahmed A $^{3^{*}}$ \\ Jaynul Islam ${ }^{4}$}

'Department of Dermatology and Venereology University of Science and Technology, Chittagong Chittagong, Bangladesh.

${ }^{2}$ Department of Gynecology and Obstetrics University of Science and Technology, Chittagong Chittagong, Bangladesh

${ }^{3}$ Department of Pathology Chattagram Maa-O-Shishu Hospital Medical College Chittagong, Bangladesh.

${ }^{4}$ Department of Histo and Cytopathology Navy Hospital, Chittagong, Bangladesh.

\section{*Correspondence to:}

\section{Professor ASM Mostaque Ahmed}

Head, Department of Pathology and Principal

Chattagram Maa-O-Shishu Hospital Medical College Chittagong, Bangladesh.

Mobile: +880-1819627349

E-mail:cmoshmc@bnslbd.net

$w w w . c m \circ s h m c j . \circ r g$

\begin{abstract}
Development of acquired idiopathic white patch on the skin is called vitiligo and around a nevomelanocytic nevus is called halo nevus. Our patient had a halo nevus in the forehead and vitiligo elsewhere. Complete remission of his vitiligo and halo nevus was achieved with topical tacrolimus ointment without incurring any harm to the patient.
\end{abstract}

Key words: Vitiligo; Halo nevus; Topical tacrolimus.

\section{INTRODUCTION}

Development of acquired idiopathic white patch on the skin and mucous membrane is called vitiligo, and around a congenital or acquired nevomelanocytic nevus is called halo nevus. Both of them could develop simultaneously in a patient ${ }^{1}$ and could be self-healing having an unpredictable course $\mathrm{e}^{2-4}$.

\section{CASE REPORT}

A boy of nine years came to us in September, 2011 with the complaint of multiple de-pigmented patches over right upper eye lid, left pina, left chest and around a congenital nevomelanocytic nevus over his right forehead. The nevus was asymptomatic and symmetric in color and shape with regular margin (Fig-1).

A full mucocutaneous examination had been done. We did not get any abnormality in him apart from the de-pigmented patches as mentioned above. However, we found low self-esteem, self-stigmatization and isolation in him and apprehension and mental distress in his parents. We took $5 \mathrm{~mm}$ punch biopsy from the nevus for histopathology and immunohistochemistry for HMB45 antigen before treatment.

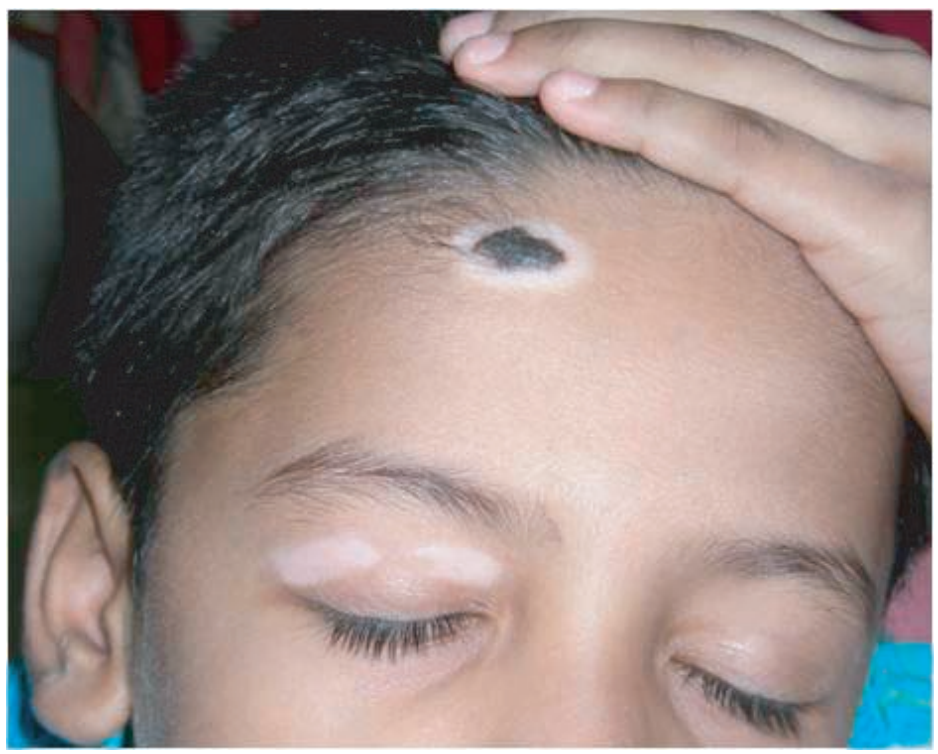

Figure 1: De-pigmented patch in and around a congenital nevomelanocytic nevus $(17 \mathrm{~mm} \times 7 \mathrm{~mm})$ over right forehead. There is a de-pigmented patch over his right upper lid. 
We treated him with tacrolimus $(0.1 \%)$ ointment. That was applied twice daily for 12 months (September, 2011-A ugust, 2012). A s it was an outpatient based treatment, we asked him to report quarterly. With our treatment, at the end of $12^{\text {th }}$ month, all his de-pigmented patches disappeared leaving behind the nevus unchanged (Fig-2).

We did not notice any side effects from this regimen. Though visible improvement was self-rewarding, we had to deliver health education in successive visits to keep his tempo high. We delivered clinical follow up quarterly for last 11 months after stoppage of our treatment. We found him in remission and fine.

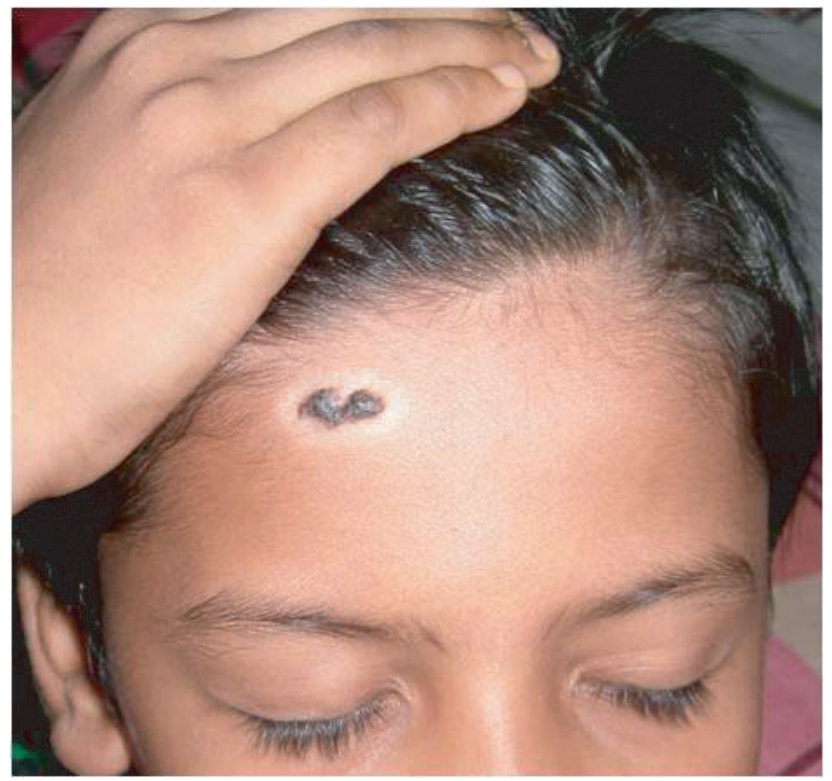

Figure 2 : Same patient, after 12 months treatment with our regimen.

Complete remission of all vitiligo patches and halo nevus is achieved with congenital nevomelanocytic nevus unchanged. The symmetry in color and shape, and border regularity of nevus is maintained. A notch in upper border is due to punch biopsy.

\section{DISCUSSION}

Our patient had halo nevus in the forehead and vitiligo elsewhere. He received topical tacrolimus ointment for vitiligo and got complete remission of both the diseases leaving behind the nevomelanocytic nevus unchanged. This is interesting and unusual in clinical practice. Halo nevus may be associated with malignant melanoma ${ }^{5}$. However in our case, a thorough clinical examination together with histopathology and immunohistochemistry of nevus excluded such an association. Ours was associated with a benign disease called vitiligo, as seen in $18-26 \%$ cases $^{6}$.

Copeman ${ }^{7}$ PWM et all found circulating antibody against the cytoplasm of malignant melanoma cell in patients with resolving halo nevus. Unfortunately we do not have facilities to assess antibody for malignant melanoma in our country. However we were able to do histopathology and immunohistochemistry. Innocent clinical appearance, and typical histopathology (Fig-3) and negative immunohistochemistry (Fig-4) helped us to recognize the case being a benign halo nevus.

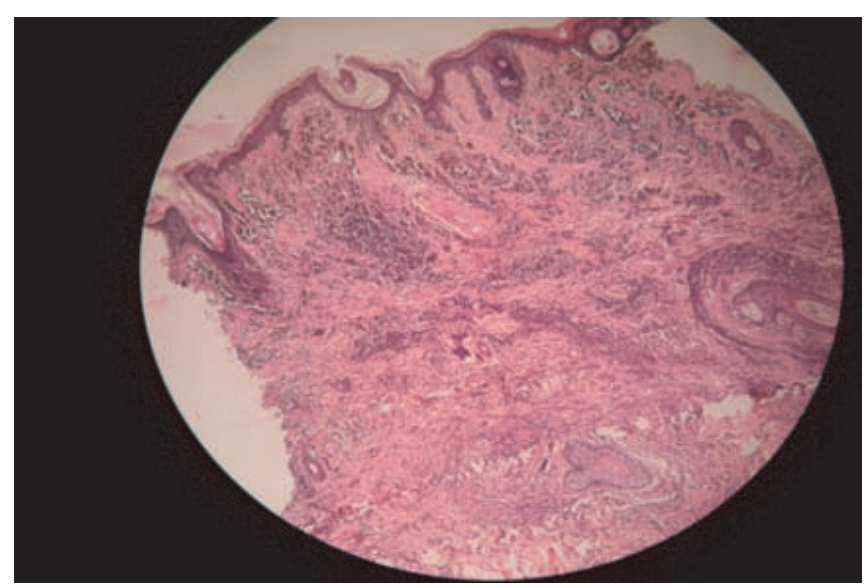

Figure 3 : Histopathology, H/E stain, 10X: A compound nevus. $N$ est of nevus cells in the dermo-epidermal junction and in the superficial and mid reticular dermis. In addition, there is Indian file arrangement of nevus cells in the dermis.

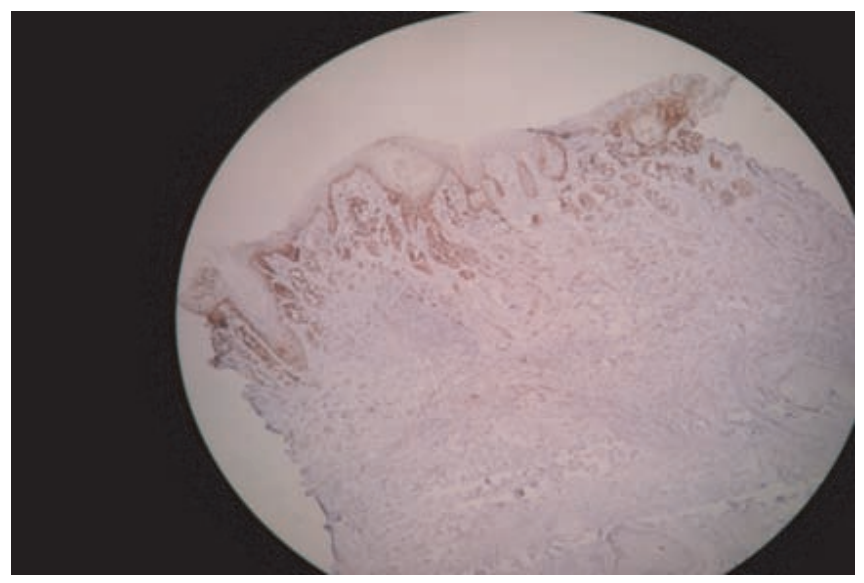

Figure 4 : Immunohistochemistry, HM B 45 stain, 10X: Positive junctional nevus cells and negative dermal nevus cells.

Some melanophages also have taken stain indicating benign nature of the nevus.

HMB45 antibody is a mouse monoclonal antibody raised against a glycoprotein present in pre-melanosome (pmel 17/ gp100), a melanoma specific marker. Fetal melanocyte, junctional nevus cells, premelanosome containing dermal melanophages and melanoma cells take this stain and become positive and thus help us in diagnostic process of diseases particularly melanoma.

In our country, white patch particularly in exposed areas is associated with intense psycho-social suffering. In that case, mere explanation and assurance as a management strategy is not enough. We have to give treatment. Our patient received treatment for vitiligo. Though the treatment of halo nevus is controversial, we brought his halo nevus under same treatment having seen its benign nature. B oth the diseases responded well and remained in remission even today (11 months after stoppage of treatment).

Histopathologically there is loss of epidermal melanocytes in the surrounding white patch ${ }^{8}$ and dense inflammatory infiltrate predominantly composed of activated T-Iymphocytes, plasma 
cells and histiocytes in the central nevus ${ }^{9}$. Treatment modalities for vitiligo work by suppressing immune response and/or stimulating the proliferation of melanocytes. Tacrolimus is a macrolide immunomodulatory and immunosuppressant agent. It inhibits T-lymphocyte activation by blocking the action of calcineurin which results in inhibition of the transcription of several cytokine genes with consequent decrease in the production of interleukins, interferon-gamma and tumour necrosis factor-alfa ${ }^{10}$. Tacrolimus ointment by exerting specific modulatory effect on cytokines might have caused repigmentation in vitiligo and halo nevus though the exact mechanism is not known.

\section{REFERENCES}

1. Itin HP, Lautenschlager S. A cquired leukoderma in congenital pigmented nevus associated with vitiligo-like depigmentation. Pediatric Dermatol, 2002; 19: 73-5.

2. Dutta AK and M andal SB. A clinical study of 650 vitiligo cases and their classification. Indian J Dermatol 1969; 14: 103-11.

3. Frank SB and Cohen JH. The halo nevi. A rchive Dermatol 1964; 89: 367-73.

4. Wayte DM and Helwig EB. Halo nevi. Cancer 1968; 22: 69-90.

5. William L. Epstein, M D; Richard Sagebeil, M D; Lynn Spitler, M D; J oseph Wybran, M D; William B. Reed, M D; M. Scot Blois. Halo nevi and melanoma. JAMA. 1973; 225(4): 373-377.

6. Kopf AW, M orril SD, Silberberg I. B road spectrum of leukoderma acquisition centrifugum. A rchive Dermatol 1965; 92: 14-35.

7. Copeman, P. W. M., L ewis, M. G., and Elliott, P. G. Immunological A ssociations of the Halo Naevus with Cutaneous M alignant M elanoma. Brit. J. Dermatol 1973; 88: 127-137.

8. Findlay GH. The histology of Sutton's nevus. BrJ Dermatol, 1957; 69: 389-94.

9. B ergman W, Willemze R, de Graaff-R eitsma C, Ruiter DJ. A nalysis of major histocompatibility antigens and the mononuclear cell infiltrate in the halo nevi. J Invest Dermatol, 1985; 85: 25-9.

10. Lawrence I. Tacrolimus (FK 506) experience in dermatology. Dermatol Ther 1998; 5: 74-84. 